\title{
Functional balance between Tcf21-Slug defines cellular plasticity and migratory modalities in high grade serous ovarian cancer cell lines
}

\section{Authors}

Sagar S Varankar1, Madhuri More, Ancy Abraham¹, Kshama Pansare³, Brijesh Kumar', Nivedhitha J Narayanan ${ }^{1}$, Mohit Kumar J olly², Avinash M. Mali', Sharmila A Bapat ${ }^{1, *}$

${ }^{1}$ National Centre for Cell Science, Savitribai Phule Pune University, Ganeshkhind, Pune 411007, India

${ }^{2}$ Center for Theoretical Biological Physics, Rice University, Houston, TX, USA

${ }^{3}$ Institute for Plasma Research \& Tata Memorial Centre, Kharghar, Navi-Mumbai, India

" Corresponding Author: Sharmila A. Bapat, National Centre for Cell Science, Savitribai Phule Pune University, Ganeshkhind, Pune 411007, India. Telephone No. +91-02025708089. Email: sabapat@nccs.res.in

The authors declare no potential conflicts of interest

(C) The Author(s) 2019. Published by Oxford University Press. All rights reserved. For Permissions, please email: journals.permissions@oup.com. 


\begin{abstract}
Cellular plasticity and transitional phenotypes add to complexities of cancer metastasis that can be initiated by single cell epithelial to mesenchymal transition (EMT) or cooperative cell migration (CCM). Our study identifies novel regulatory cross -talks between Tcf21 and Slug in mediating phenotypic and migration plasticity in high-grade serous ovarian adenocarcinoma (HGSC). Differential expression and sub-cellular localization associate Tcf21, Slug with epithelial, mesenchymal phenotypes respectively; however gene manipulation approaches identify their association with additional intermediate phenotypic states, implying the existence of a multi-step epithelial-mesenchymal transition program. Live imaging further associated distinct migratory modalities with the Tcf $21 /$ Slug status of cell systems and discerned proliferative/passive CCM, active CCM and EMT modes of migration. Tcf21-Slug balance identified across a phenotypic spectrum in HGSC cell lines, associated with microenvironment induced transitions and the emergence of an epithelial phenotype following drug exposure. Phenotypic transitions and associated functionalities following drug exposure were affirmed to ensue from occupancy of Slug promoter E-box sequences by Tcf21. Our study effectively provides a framework for understanding the relevance of ovarian cancer plasticity as a function of two transcription factors.
\end{abstract}

\title{
Summary
}

Our study implicates Tcf21-Slug in HGSC cellular plasticity, provides a functional comprehension of phenotypic transitions in response to extrinsic stimuli and effectively captures distinct migratory modalities resulting from these transitions with a real time imaging assisted protocol.

Keywords: Plasticity; Tcf21; Slug; sub-cellular localization; migratory modalities

\section{Abbreviations:}

aCCM, active collective cell migration; BMP7, bone morphogenetic protein 7; $\mathrm{CCM}$, collective cell migration; Cdh1, E-cadherin; E, epithelial; EM, epithelial- mesenchymal hybrid; EMT, epithelial to mesenchymal transition; HGSC, high grade serous carcinoma; $i E$, intermediate epithelial; iM, intermediate mesenchymal; $\mathrm{K} / \mathrm{O}$, knock-out; $\mathrm{M}$, mesenchymal; $\mathrm{Nn}$, nearest neighbours; OE, over-expression; PCA, principal component analysis; $\mathrm{PCCM}$, passive collective cell migration; SS, serum starved; TCGA, The Cancer Genome Atlas; TF, transcription factors; TGF $\beta$ transforming growth factor $\beta$ Vim, vimentin. 


\section{Introduction}

Intrinsic cellular identities originate during embryonic commitment and are fated for tissue specific spatio-temporal roles through cross-talks with niche components (1). Destabilization of homeostasis under pathological conditions alters the tissue milieu to revoke inherent cellular plasticity and restore primitive phenotype(s), architecture and functions to facilitate disease progression $(2,3)$. Co-operative action of regulatory molecular circuits such as the role of Slug-S ox9 in maintenance of mammary basal and luminal epithelial cells, are diverted in aberrant contexts like cell transformation to redefine cell fates $(4,5)$. Innate capabilities and perturbed microenvironment thus align as distinct etiologies in cancer $(6,7)$. Previous studies in epithelial ovarian carcinomas (EOC) identified S tem-A, Stem-B, E pi-A, E pi-B and Mes subtypes that correspond with epithelial $(E)$, intermediate epithelial (iE), intermediate mesenchymal (iM) and mesenchymal (M) phenotypic features (8,9); a subsequent study reported $\mathrm{iE}$ and $\mathrm{iM}$ as extensions of an epithelial-mesenchymal hybrid state $(\mathrm{E}-\mathrm{M})$. Furthermore, comprehensive analyses of the most aggressive EOC viz. high-grade serous adenocarcinomas (HGSC) have revealed diverse molecular signatures that correlate with epithelial/differentiated (E/D), mesenchymal $(M)$, proliferative $(P)$, immunoreactive (IR) features and require adoption of differential therapeutic strategies (10-13). Hence, the disparate collation of EOC datasets can misrepresent the clinical associations of tumor subtypes with phenotypic states and should be avoided to improve disease management.

Our previous analyses of HGSC expression datasets in The Cancer Genome Atlas (TCGA) resolved three molecular sub-classes associated with discrete mechanisms of metastases. Class1 exhibited co-operative cell migration (CCM), Class2 associated with epithelialmesenchymal transitions (EMT) while Class3 presented with mixed features $(11,14)$. Correlating with reports using the same datasets suggested CCM-Class tumors to present as $\mathrm{P} / \mathrm{D} / \mathrm{IE}$ sub-class, EMT-Class tumors as M/R/M while the heterogeneous Class 3 tumors possibly represent IR/D phenotypes. While molecular signatures for HGSC subtypes have been defined across multiple studies, the limited comprehension of aberrantly activated plasticity programs curb effective translation of previous findings. In the present study we discern HGSC cell phenotypes (E/M/intermediates) as a function of Tcf21 and Slug expression, localization and activity. Functional evaluation assisted by real-time imaging, associated phenotypic states with either EMT, active CCM or proliferative (annotated as passive (CM / pCCM) modes of wound closure. As opposed to the rigidity of epithelial / mesenchymal phenotypes, intermediate states exhibited transitions between EMT and, aCCM (but not pCCM) modalities and responded with enhanced plasticity in presence of 
positive/negative growth regulators as a function of altered Tcf21-Slug sub-cellular localization. Strikingly, emergence of epithelial features involved transcriptional repression of Slug by Tcf21. Our study thus, highlights the antagonistic interplay of Tcf21-Slug in maintenance of distinct cellular phenotypes and presents them as potential biomarkers for phenotype assisted tumor stratification.

\section{Materials and Methods}

\section{Cell Culture}

Ovarian cancer cell lines used in the study were all of the high grade serous histology $(15,16)$. A4 cells were previously established in our lab from ascites of a HGSC patient (17), OVCAR3 cells were obtained from the cell repository at NCCS, Pune, India. Dr. S. Mok (M. D. Anderson Cancer Center, Texas, USA) and Prof. Viktor Magdalen (Klinische Forschergruppe der Frauenklinik der TU München) provided OV90 and OVMZ6 cell lines, respectively. OVCA420, PEO14, OVCA432 and CAOV3 cells were provided by Prof. Judith Clements (Translational Research Institute, Australia). OVCAR3, OVCA432, CAOV3, OV90, OVCA420 and PEO14 cells were grown in RPMI 1640 (Gibco, Waltham, Massachusetts, USA) supplemented with $10 \%$ fetal bovine serum. A4 cells were cultured in Minimal Essential Medium / MEM (Gibco, Waltham, Massachusetts, USA) supplemented with 5\% fetal bovine serum and $1 \%$ non-essential amino acids; Dulbecco's Modified Essential Medium / DMEM (Gibco, Waltham, Massachusetts, USA) supplemented with 5\% serum, $100 \mu \mathrm{M}$ asparagine (Sigma, St. Louis, Mis souri, USA) and $100 \mu \mathrm{M}$ arginine (Sigma, St. Louis, Missouri, USA) was used for culturing OVMZ6 cells. All cell lines were maintained in a humidified incubator at $37^{\circ} \mathrm{C}$ under a $5 \% \mathrm{CO}_{2}$ atmosphere. Cell lines were authenticated by Short Tandem Repeat Profiling and comparison with ATCC and EACC databases (J une 2018).

\section{Transfection, Generation of TF Derivative Clones}

For generation of knockouts via genomic editing OVCAR 3 and A4 cells were transfected with 500ng of commercially available CrispR_GFP vectors against Tcf21 and Slug (Sigma, St. Louis, Missouri, USA) respectively using Lipofectamine 3000 (Invitrogen, Carlsbad, California, USA) as per the manufacturer's protocol. Post transfection, cells were incubated at $30^{\circ} \mathrm{C}$ for 48 hours to ensure optimal CrispR activity that was confirmed by the T7 endonuclease assay (data not provided). Single cell sorting of GFP positive cells was performed with BD Aria II SORP into 96 well plates. Sorted cells were allowed to grow for clone establishment and subjected to molecular (qPCR, immunoblotting, immunofluorescence staining) and functional (in vitro wound closure) validation. Successful 
knockouts (E2 - Tcf21 knockout, D7 - Slug knockout) obtained from CrispR transfections, were further transfected with GFP vectors for Slug and Tcf21 respectively, sorted to obtain single cell clones and validated. $\mathrm{T} c f 21^{\mathrm{K} / \mathrm{S}} \mathrm{Slug}{ }^{\mathrm{OE}}$ clones could not be established due to rapid apoptosis.

\section{Treatments}

Cells were treated as follows prior to R NA extraction and immunofluorescence staining:

Serum Deprivation: Cells were allowed to grow for 24 hours post seeding, following which spent medium for each cell line was replaced with equal volume of serum free media as indicated for each cell line. Growth Factor Exposure: 24 hours following serum deprivation, cell lines were exposed to either BMP7 $(10 \mathrm{ng} / \mathrm{mL}), T G F \beta(10 \mathrm{ng} / \mathrm{mL})$ or a combination of both (Invitrogen, Carlsbad, California, US A). Paclitaxel Treatment: Cells were allowed to grow for 24 hours post seeding, after which spent medium for each cell line was replaced with equal volume of complete medium containing IC 50 concentrations of paclitaxel (Sigma, St. Louis, Missouri, USA) optimized for each cell line (IC 50 values for each cell line are available on request). $0.1 \%$ DMSO was used as a vehicle control.

\section{Derivation of TF network, correlation and expression analys is of TFs and phenotypic markers in TCGA-HGSC dataset}

Details are provided in Supplementary Material.

\section{RNA extraction, CDNA synthes is, RT-PCR and $q P C R$}

Trizol (Invitrogen, Carlsbad, California, USA) based total RNA extraction was achieved from $80 \%$ confluent cultures of cell lines following respective treatments as previously described (18). Details are provided in SI Material along with list of primers.

\section{Immunos taining, image acquisition and quantification of signal intens ity:}

Immunostaining was performed as previously described (18). Quantification of signal intensities for transcription factors was performed with the LAS-AF software (Leica Microsystems, Wetzlar, Germany). Briefly, ROIs were defined from phase contrast images around nuclear margins (for TFs) or cell boundaries (for Cdh1, Vim) of each cell followed by extraction of signal intensities for Hoechst and respective fluorescent channels. Relative nuclear signal intensity for transcription factors was determined as a ratio of TF-/Hoechstassociated fluorescence, while C dh1,Vim were normalized to unstained (primary antibody control) samples to eliminate non-specific signals obtained due to the staining protocol. 
Quantification was performed for a minimum of 50 nuclei for each sample. List of antibodies used is provided in Supplementary Material.

\section{Immunoblotting:}

Immunoblotting protocols were followed as previously described (18). List of antibodies used is provided in Supplementary Material.

In vitro wound healing assay, live cell imaging, processing and quantification of data, PCA for migration

Wound healing assay was performed as per previous protocols (14). Under specific experimental conditions media additives were administered including $5 \%$ serum, TGF $\beta$ $(10 \mathrm{ng} / \mathrm{ml})$, BMP7 $(10 \mathrm{ng} / \mathrm{ml})$ or a combination of both growth factors. The wound was allowed to heal and was monitored up to 144h. Images were captured on Olympus IX71 microscope and analyzed by the T-S cratch Software (Tobias Gebäck and Martin Schulz).

For live cell imaging, images were acquired for 30 hours, following infliction of wound on a cell monolayer, on an inverted laser scanning confocal fluorescence LPSS 5 microscope maintained at $37^{\circ} \mathrm{C}, 5 \% \mathrm{CO}_{2}$ atmosphere (Leica Microsystems, Wetzlar, Germany). In case of specific treatments, additives were added as previously described. For live cell imaging of paclitaxel treated cells, drug treatment for 48 hours was followed by harvesting of residual cells and seeding for wound healing assay. In the absence of additives live cell imaging was performed in serum free media in the presence of $20 \mathrm{ng} / \mathrm{mL}$ of mitomycin C" (Sigma, St. Louis, Missouri, USA). Images captured for live cell imaging were exported from the LAS AF software (Leica Microsystems, Wetzlar, Germany) with .avi extensions. For elimination of shadow effects in images avi files were opened in Fiji software, duplicated, inverted and averaged with original image using the Image Calculator. Processed files were subjected to thresholding to obtain binary images which were eventually processed through MTrack2 plugin to obtain $X$ and $Y$ co-ordinates for all the cells detected in the microscopic field of interest. Positional co-ordinates over a period of 16 hours was used to calculate mean displacement and velocity for each cell, however, cells lacking this data at any time point were excluded from further analysis. Furthermore, binary images were processed through the BioVoxxel plugin to determine the mean number of nearest neighbors ( $\mathrm{Nn}$ ) around each cell particle. One cell diameter was used as a threshold to identify $\mathrm{Nn}$ around each cell as depicted in Supplementary Figure S2. Mean displacement, velocity and number of nearest neighbors were analyzed in matlab2013b (Mathworks) with an open source code available for principal component analysis. First two components capturing maximum variance of the data were represented using SigmaPlot 10.0 software (SPSS). 


\section{Boyden chamber invasion assay}

Trans-well invasion assays were performed, as previously described (19).

\section{Suspension culture ass ses ment}

Suspension cultures for HGSC cell lines were generated as described by earlier studies (20).

\section{FACS sorting and flow cytometry}

FACS and Flow cytometry were performed as previously described (20). List of antibodies used is provided in Supplementary Material.

\section{Cloning, expression and purification of GST-Tcf21 protein}

Human Tcf21 cDNA was amplified from A4 cells, cloned into pGEX-6P-1 GST vector and transformed into Escherichia coli BL21 codon Plus (Agilent Technologies, Santa Clara, California, USA). Details of cloning and protein purification are provided in SI Materials and Methods.

\section{Electrophoretic mobility shift as say (EMSA)}

EMSA was performed as previously described (18).

\section{Chromatin Immunoprecipitation Ass ay and ChIP-PCR}

ChIP was performed as described earlier (19); ChIPped DNA amplified using the GenomePlex Complete Whole Genome amplification kit as per manufacturer's instructions (Sigma, St. Louis, Missouri, USA). Specific primers were used to amplify E-boxes within promoter regions of genes, amplified products run on $1.5 \%$ agarose gels, and band intensities measured by densitometric analysis using Genetool3.6 (Syngene, Bengaluru, India). List of antibodies and primers used is provided in S upplementary Material.

\section{Luciferase Cons tructs and Luciferase as say}

Luciferase constructs for E-cadherin and Slug wild type promoters along with deletion mutants $\triangle S 1-S 2$ were previously generated in the pGL3 luciferase plasmid (18). All constructs were verified by sequencing. $0.5 \times 10^{5}$ OVCAR3, A4, and derivatives were transfected with $0.5 \mu \mathrm{hg}$ Luciferase reporter plasmids control and E-cadherin/S lug promoter containing plasmids in 24-well plates using Lipofectamine 3000 (Invitrogen, Carlsbad, California, USA). Renilla luciferase was added (10 $\mathrm{ng})$ to each transfection as control. 
Luciferase activity was measured using a Dual Luciferase assay kit (Promega, Madison, Wisconsin, USA) either post transfection with pE GFP-Tcf21 and/or post paclitaxel treatment. Empty $\mathrm{pEGFP}$ and $\mathrm{pGL} 3$ vectors were used as controls.

\section{Quantitative and S tatis tical Analys is:}

Pearson's Correlation Co-efficient across the TCGA-HGSC datasets was calculated using the GraphPad Prism 6.0 software. Significant differences in gene expression profiles across the HGSC cell line panel were calculated by One-Way ANOVA using the Sigma Stat 3.5 Software. Significant differences following treatments in gene expression profiles, invasive capabilities and wound healing properties of HGSC cell lines and derivative clones, and luciferase activity for promoter assays were calculated by paired Student's t-test using the Sigma S tat 3.5 Software. Similarly, ChIP binding efficiencies were calculated by un-paired Student's t-test. Differences in nuclear intensities of Tcf 21 , Slug, $C$ dh1 and Vim were calculated by Tukey's Test using the Sigma Stat 3.5 Software. Differences were considered significant if $p<0.05$.

\section{Results}

\section{Tcf21 and Slug expression governs a multi-s tep phenotypic axis in HGSC}

Previous data (14) identified class-specific expression of thirteen transcription factors that were examined in the present study for association with distinct phenotypic states in HGSC (TFs; S upplementary Table S1); immunomodulatory TFs AIF 1,ETV7 were excluded from further analysis due to limitations of our experimental systems. Promoter occupancies between the remaining TFs, derived from consensus DNA binding sequences, predicted a cross-regulatory network that included $10 \mathrm{TFs}$ from Class1 (CCM/epithelial tumor class) viz., Tcf21, Sall2, Peg3, Gata4, Kcnip3, Znf219, Foxl2, Gli1, Sox12, Tcf7l1 and one TF viz., Slug from Class2 (EMT/mesenchymal tumor class; Figure 1a, Supplementary Figure S1a; details of TF network generation are provided in Supplementary Material) Correlation analysis amongst these TFs predicted co-regulation of Tcf21, Gata4, Peg3, Gli1, Foxl2 that correlated positively with each other and negatively with Snai2 (Slug) in the TCGA dataset (S upplementary Figure S1b). Profiling these TFs in class-representative cell lines robustly associated CCM-Class (OVCAR3 cells) with Tcf21 and EMT-Class (A4 cells) with Slug expression; however, the lack of a strong correlation between mRNA and protein profiles limited their further validation (Figure 1b, Supplementary Figure S1c). Thus, molecular profiles in our cell line models and previous association of Tcf21, Slug with the epithelial, 
mesenchymal phenotypes, respectively $(19,21)$, prompted our focused efforts on discerning their role in cellular plasticity of ovarian cancer cell lines.

Towards ascertaining the role of Tcf21 and Slug in phenotype maintenance, we further derived knockout $(\mathrm{K} / \mathrm{O}$ ) clones of the dominant TF (genomic editing by commercial CrispR constructs) followed by over-expression (OE) of the other TF in parental cell lines OVCAR3 (Tcf21 ${ }^{\text {native }}$ ) and A4 (Slug ${ }^{\text {native }}$; Supplementary Fig. S1d); stable knockouts were affirmed by sequencing (data not shown). Molecular profiles revealed high Tcf21, E-cadherin (Cdh1) expression in OVCAR 3 cells whereas $\operatorname{Tcf} 21^{\mathrm{K} / \mathrm{O}}(\mathrm{E} 2)$ exhibited high nuclear Slug and low Cdh1. A4 cells expressed high Slug, Vimentin (Vim) with low Cdh1, however, sustained expression of other EMT-TFs (Zeb1/2,Twist1,S nail,Prrx1) in the Slug ${ }^{k / 0}$ (D7) led to minimally altered Cdh1 and Vim despite enhanced nuclear Tcf21 localization (Figures 1c,1d). Interestingly, A4_Slug ${ }^{\mathrm{K} / \mathrm{T}} \mathrm{Tcf} 21^{\mathrm{OE}}$ (TCF_OE) cells exhibited reduced expression of EMT-TFs and correlated with enhanced Cdh1 whilst retaining Vim expression (Figures 1c,1d). High luciferase activity in OVCAR3 and TCF_OE cells (reporter assay for Cdh1 promoter harboring Slug binding E-box sequences), and reduced activity in E2, A4 and D7 cells suggested phenotypic variability as a function of Tcf21 and Slug expression (Figure 1e). Functional validation identified differential rates of wound closure between parental and derivative clones to be positively influenced by serum (Figure 1f). In the absence of serum, OVCAR 3 and E 2 cells failed to induce wound closure, suggesting the absence of functional EMT despite enhanced Slug and reduced Cdh 1 levels in E2 cells; wound healing efficacy was drastically curbed in TCF_OE as compared to A4 and D7 cells. Derivative clones, thus represented intermediate phenotypes along the epithelial-mesenchymal ( $E-M)$ axis wherein OVCAR3 $\left(T c f 21^{\text {native }}\right)$ and E $2\left(T c f 21^{\mathrm{k} / 0}\right)$ exhibited a rigidity towards functional phenotypic

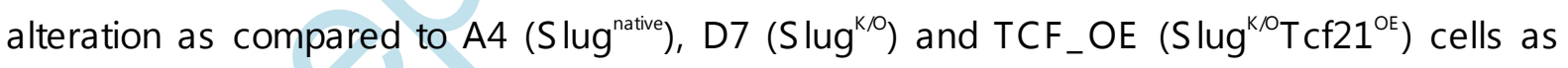
indicated by molecular and functional readouts (Figure $1 \mathrm{~g}$ ). These findings whilst associating Tcf21, Slug with epithelial, mesenchymal phenotypes respectively, highlight the inability of these TFs towards inducing a bilateral phenotypic switch and possibly imply E-M transitions as a multi-step process.

\section{Tcf21-S lug status of HGSC cells associates with differential modes of migration}

A disconnect between wound closure efficacies and expression of phenotypic markers in derivative clones prompted the adoption of high-resolution approaches to investigate their respective migratory modalities. Time lapse microscopy imaging improved the in vitro wound healing assay by enhancing the visual output. OVCAR3 and E 2 cells exhibited proliferationdriven cell displacement that we termed passive CCM ( $\mathrm{PCCM}$ ), A4 mediated rapid wound 
healing through a combination of single cell and active CCM (EMT and aCCM respectively) while its derivatives D7, $\left(\mathrm{S} \mathrm{lug}^{\mathrm{K} / \mathrm{O}} \mathrm{T} \mathrm{cf} 21^{\mathrm{OE}}\right.$ ) exhibited aCCM exclusively (Supplementary Video S1). Cell position co-ordinates during wound closure allowed quantification of these processes by virtue of migration trajectories, mean cell velocity and nearest neighbors ( $\mathrm{Nn}$; Supplementary Figure S2). pCCM driven wound closure was associated with minimal cell displacement, low migratory velocity and a high $\mathrm{Nn}$; aCCM was defined by moderate displacement / velocity and high Nn while EMT cells exhibited high displacement / velocity with low Nn (Figures 2a-2c). Serum addition enhanced displacement, velocity and reduced $\mathrm{Nn}$ in aCCM and EMT phenotypes while PCCM cells increased Nn by virtue of extensive cell proliferation. Presence of mitomycin ' $C$ ', a proliferation inhibitor, in the absence of serum distinguished cell displacement attributed by active migration (aCCM/EMT) as opposed to cell division driven passive movement ( $\mathrm{pCCM}$ ). Principal component ( $P C$ ) analysis performed with these datasets supported differential migratory modes wherein PC1 (variance between velocity and displacement vs. Nn) and PC2 (variance between displacement vs. velocity) effectively resolved cell lines associated with PCCM, aCCM and EMT-aCCM modes of migration while capturing serum induced shifts in respective modalities (Figure 2d). Differential $\mathrm{Nn}$ frequencies between A4 and D7 proved a useful metric for distinguishing EMT from aCCM apart from the visualization in real time imaging. While visual outputs affirmed association of Tcf21 and Slug with distinct migratory modes in the derivative clones, PCA permitted an un-biased segregation of these modalities as a function of TF expression. This gradient of migration modalities effectively agrees with the artificial $\mathrm{E}$ to $\mathrm{M}$ spectrum depicted in Figure $1 \mathrm{~g}$, emphasizing rigidity of the $\mathrm{E}$ phenotype, and supporting the absence of biphasic phenotypic switches in HGSC.

\section{Tcf21 is a transcriptional repres s or of Slug}

In view of the predictive TF network and inverse associations in our derivative clones, we probed possible cross-regulation between Tcf21 and Slug. Luciferase activity (reporter assay for SNAI2 promoter harboring Tcf21-binding consensus E-box sequences) was severely reduced in A4 cells exogenously over-expressing Tcf21 (Figures 3a,3b). In vitro binding assays also affirmed physical interactions between recombinant Tcf21 and S1,S3, S5 SNAI2 E-boxes. Further probing of Tcf21-bound chromatin complexes in OVCAR3 cells through immunoprecipitation indicated affinity for S2,S3,S6 SNAI2 E-boxes (Figures 3c,3d). Similar regulation of Tcf 21 expression by Slug was not observed. Varied Tcf 21 binding affinities in cell-free and cell-based systems emphasize the significance of cellular context in target recognition and imply possible involvement of other co-regulatory factors in the process. 
These findings suggest transcriptional repression of Slug by Tcf21 towards maintenance of epithelial properties in HGSC. 


\section{HGSC tumors and cell lines intrinsically display a spectrum of phenotypes and modes of migration}

Towards resolution of distinct phenotypes in HGSC at steady state and describing E-M transitions as a multi-step process, we primarily analyzed the heterogeneity of phenotypic markers in TCGA-HGSC dataset. Correlation analys is of gene expression data for epithelial and mesenchymal markers generated four clusters (C1-C4) representative of specific phenotypic attributes (Supplementary Figure S3a). C1 genes included components of epithelial cell junctions and keratins, C2 genes associated with mesothelial and myoepithelial phenotypes across several tissues (breast, ovary, peritoneal linings) (22-24), C3 comprised of cell junction molecules encountered in mesenchymal cells (10), while C4 included genes commonly expressed in the endothelial and stromal lineages $(25,26)$. Apart from segregation of these gene groups, correlation analysis identified a heterogeneity of phenotypic markers in HGSC which could be indicative of existing diverse phenotypes. Hierarchical clustering of known epithelial and mesenchymal TFs in this data yielded five distinct tumor groups (Supplementary Figure S3b). Two of these were exclusively epithelial $(E)$ and mesenchymal $(M)$ while a third was considered $E-M$ by virtue of a heterogeneous double-positive signature. A separate cluster identified as intermediate $E$ (iE), expressed $E$ markers, Snail, Twist1 but lacked Tcf21, while the tumor group with low EMT-TF expression was assigned as intermediate $\mathrm{M}(\mathrm{iM})$.

In vitro validation of identified phenotypic states proceeded via parallel screening of $E, M$ markers and TF transcripts across a panel of HGSC cell lines. Molecular profiles resolved five phenotypic states through definitive correlations between $E$ ( $C$ dh1, Ck19), M (Vim,FAP) markers and TFs (Tcf21, Slug and Prrx1 is oforms $1 \mathrm{a}$ and $1 \mathrm{~b}$; S upplementary Figures S3cS3e; Prrx1a, Prrx1b transcripts - P1a, P1b respectively, protein represented as Prrx1) (27). Non-availability of isoform-specific Prrx1 antibodies restricted further definitive studies for this TF. OVCAR3, OVCA432 (high E, nuclear Tcf21, low P1a-P1b-nuclear Prrx1, low nuclear Slug) and OVMZ6 cells with an inverse profile defined the $E$ and $M$ ends of the spectrum. CAOV3 and OV90 were identified as iE (high E, FAP, nuclear Tcf21, low P1a-P1b-moderate nuclear Prrx1, low nuclear Slug), OVCA420 and PEO14 as E-M (E, M markers, all TFs in nucleus) and A4 as iM phenotype (CK19, M markers, low cytoplasmic Tcf21, high P1b, nuclear Prrx1 and Slug). These phenotypes could be perturbed by external stress such as serum deprivation that induced $\mathrm{Cdh} 1$ and nuclear Tcf21 expression, concomitantly decreasing Slug, FAP and Vim in the cell line panel to shift the spectrum towards the $E$ phenotype (Figures 4a,4b). 
Probing phenotypic - functional associations assigned spheroid generation capabilities to intermediate states while $\mathrm{E}$ and $\mathrm{M}$ cells formed clumps/aggregates (Figure 4c). Except the $\mathrm{E}$ state, all cell lines were highly invasive in response to a serum gradient (Figure $4 \mathrm{~d}$ ). Differential migration capabilities were evident wherein A4, OV90 and OVCA420 cells filled the open wound area in absence of serum as opposed to $\mathrm{E} / \mathrm{IE}$ and surprisingly $\mathrm{M}$ cells that required serum to achieve wound closure (Figures 4e.i,4e.ii). Live imaging and PC analyses based on pre-defined quantitative metrics revealed pCCM in E and $i E$, aCCM in $E-M$, coupling of aCCM-EMT in iM and EMT in M cells (Supplementary Video 2; Figure 4e.iii). Interestingly, serum deprivation led to nuclear localization of Slug in a few E-M state cells that possibly induces rare EMT events visualized in live imaging. Thus, the cell line panel reaffirmed existence of a phenotypic spectrum in HGSC defined by inherent Tcf21-Slug profiles and captured cellular plasticity as intermediate states following growth factor deprivation.

\section{Micro-environment influenced phenotypic inter-conversions are captured by nuclear Tcf21 and Slug expression}

Further examination of cellular plasticity across the E-M axis and phenotype specific association of Tcf21-Slug, proceeded with exposure of the cell line panel and derivative clones to growth factors known to mediate directed phenotypic changes. TGF $\beta$-BMP signaling plays a crucial role during ovarian/fallopian tube development and homeostasis. Additionally, the antagonistic influence of TGF $\beta 1$ and BMP7 on epithelial and mesenchymal phenotypes make them suitable triggers to assess HGSC cell line plasticity. E and iE cells were refractory in their response to both growth factors; BMP 7 induced Cdh1, nuclear Tcf21 and reduced Slug levels whereas TGFß1 exposure up-regulated Slug concomitantly with reduced Cdh1 and Tcf21 in E-M, iM and M cells (Figure 5a, Supplementary Figures S4a, $S 5 a$, S6). Dominant effects of BMP7 over TGFß1 were observed in E-M and iM cell lines (high C dh1, nuclear Tcf21, low Slug) when the growth factors were provided in combination. Similarly, exposure of derivative clones (E2, D7 and TCF_OE) and parental cell lines (OVCAR3, A4) generated distinct responses that correlated with an inherent Tcf21-Slug balance. OVCAR 3 cells were refractory to both growth factors while $\mathrm{E} 2$ responded poorly to BMP7 and exhibited enhanced nuclear Slug expression following exposure to TGFß1. A4, D7, TCF_OE cells responded to BMP7 through nuclear localization of Tcf21, enhanced Cdh1 expression and reduced Slug. Conversely, TGF $\beta 1$ repressed Tcf21, Cdh1 in A4 and D7 cells with concomitant increase in Slug and Vim expression while TCF_OE responded poorly to the same (Figure 5a, Supplementary Figures S4b, S5b, S6). Thus, clone 
responses to combination of these two growth factors agreed with observations in the cell line panel. Integrating Tcf21 and Slug transcript profiles across all cell lines and derivatives under varying culture conditions (steady-state, serum-deprivation, growth factor exposure) with TF-promoter binding studies aided the generation of a mathematical model that identified TCF $21^{\text {high }}$-SLUG ${ }^{\text {low }}(E), T C F 21^{\text {low }}-S L U G^{\text {high }}(M)$ and TCf $21^{\text {mod }}-S$ lug ${ }^{\text {mod }}(E-M)$ as stable phenotypes while $\mathrm{iE}$ and $\mathrm{iM}$ were depicted as metastable states associated with phenotypic switches. Drastic fluctuations in Tcf21 and Slug expression correlated with transitions at the $\mathrm{E}$ and $\mathrm{M}$ ends respectively (S upplementary Figure S4c).

Functional evaluation of the micro-environmental influences revealed rate of wound closure to be restricted by BMP7 and enhanced by TGF $\beta$ in E-M, iM and $M$, while minimal changes were evident in $\mathrm{E}$ and $\mathrm{iE}$ cells (Figure $5 \mathrm{~b}$ ). Combination treatment with both cytokines revealed a dominant effect of BMP 7 over TGF $\beta$ in hindering wound closure. This functionality was altered in derivative clones wherein E2 exhibited moderately enhanced wound closure following exposure to TGF $\beta$ with minimal response to BMP7 in agreement with altered Tcf21Slug balance; TGF $\beta$ enhanced and BMP7 reduced wound closure in D7 cells whereas TCF_OE exhibited minimal alterations. These observations assign cellular rigidity to $E, M$ and plasticity to intermediate states. Conclusively, Tcf21-Slug expression and sub-cellular localization correlates with cellular states across the spectrum and are possible determinants of functionality in migration and phenotypic plasticity.

\section{Drug induced plasticity in HGSC as sociates with Tcf21-Slug crosstalk}

Phenotypic plasticity in the E-M spectrum was further assessed in response to a chemotherapeutic challenge (paclitaxel) and evaluated as a function of Tcf21-Slug expression. PC analysis of live migration derived metrics identified a switch from EMT to aCCM mode for $M$ and $\mathrm{M}$ cells after treatment (maximum variance along both $\mathrm{PCS}$ ), $\mathrm{E}-\mathrm{M}$ and $\mathrm{iE}$ adhered to their characteristic modes of migration albeit at reduced velocities (moderate variance along both PCs) while PCCM phenotype in E cells was unaltered (Figure 6a, Supplementary Video S3). These altered migratory metrics across the spectrum were associated with increased nuclear Tcf21 concurrently with reduced Slug expression (Figure 6b). Pacliaxel exposure induced an iE phenotype in $E$ and $i E$ cell lines, a similar shift towards the $\mathrm{E}$ phenotype was evident in $\mathrm{E}-\mathrm{M}$, $\mathrm{iM}$ and $\mathrm{M}$ cells although the extent of each shift was distinct To assign a mechanistic understanding to drug-induced phenotypeswitching, we probed occupancy of Tcf21 at the Slug promoter as a read-out of the $E$ phenotype. Enhanced affinity of Tcf21 for the S2, S3 E-boxes in SNAI2 promoter was identified following paclitaxel treatment and further complemented by negligent luciferase 
activity in Tcf21 over-expressing paclitaxel treated A4 cells (Figures $6 c, 6 d$ ). Deletion of S2 Ebox from the Slug promoter dramatically curbed Tcf21 mediated Slug repression in the luciferase assay. These results together identify a Tcf21-driven phenotypic switch in HGSC cells that results in the emergence of epithelial traits.

\section{Discussion}

The anatomical proximity of ovarian surface and FT epithelium as sites of perturbed epithelial integrity, following ovulation and wound healing, have led to conflicting opinions on the cell-of-origin of ovarian cancer $(14,28,29)$. The present study, restricted to the most aggressive subtype viz. HGSC, identifies Tcf21-Slug cross-talk in maintenance of intrinsic cellular states, wherein Tcf21-mediated Slug repression emerged as a feature of the epithelial phenotype. While previous studies exclusively implicate Slug as an EMT-TF (30), Tcf21 exhibits a tissue-specific activity in facilitating E-M transitions. In the context of cardiac development, Tcf21 expression is linked with the onset of EMT in endocardial progenitors towards sculpting of the cardiac cushion and valves (31). Conversely, repression of this TF in renal and lung carcinomas results in overt metastasis, which can be suppressed through the activation of Kiss1, a tumor suppressor gene and target of $\operatorname{Tcf} 21(32,33)$. A balance of these TFs was associated with cellular plasticity as gauged by molecular and functional responses to extrinsic stimuli. Cells with native Tcf21 were conferred a highly rigid epithelial phenotype whereas dual nuclear positivity (Tcf21 and Slug) was associated with enhanced plasticity. Rigidity of epithelial and mesenchymal states was challenged by deriving intermediates $(\mathrm{iE}, \mathrm{E}-\mathrm{M}, \mathrm{iM})$ and correlating phenotypes with discrete modes of migration despite comparable invasion (Supplementary figure S7a). The latter is thus underscored as a universal feature in HGSC and uncoupled from EMT (34).

Differential migration recapitulates events at the tumor edge $(35,36)$, wherein destabilization of the tissue architecture by a degradative secretome and altered mileu may promote EMT, while exertion of tensile forces by adherens junction-linked acto-myosin complexes may induce CCM allowing invasion/deposition of tumor cells in the peritoneum (37-42). Our quantification of in vitro metrics, distinguished discrete migratory modalities across the phenotypic spectrum and correlated them with Tcf21-Slug expression. Application of live cell imaging also enhanced resolution of the in vitro wound healing assay, thus generating an improved informative method. Exploring the physiologically relevant BMP7-TGF $\beta$ signaling dynamics during ovulation (43-45), we identified the imposition of reduced migration with induction of nuclear Tcf21 and epithelial features through BMP7 exposure, while TGF- $\beta$ 
enhanced EMT/ACCM mediated migration and mesenchymal features triggered by Slug expression (Supplementary Figure S7b). While TGF $\beta$ has been extensively associated with HGSC pathology $(46,47)$, previous reports on BMP7 have indirectly implied it's role in therapy resistance, tumour progression and inhibition of TGF $\beta$ signaling in ovarian cancer (48-50). However, our findings conclusively identify a reciprocal role for these cytokines in governing cellular plasticity of ovarian cancer cells, which is reminiscent of their physiological activity during ovulation (45). These findings may be extrapolated to derive similarities between EMT-aCCM cooperation and OSE wound healing, while maintenance of the rigid, disparate epithelial state during pCCM could be akin to the FT-p53 signature-associated precursor lesions $(51,52)$. Serum withdrawal as well as paclitaxel exposure promoted a Tcf21 driven epithelial state and induced aCCM that could represent a pro-survival mode of co-operative drug resistance. Moreover, Tcf21 expression associated with reduced sensitivity, and in return higher IC $_{50^{\prime}}$ of HGSC cell to the drug when tested across the HGSC panel (data not shown). Paclitaxel-induced shifts were further associated with Slug repression via direct promoter binding by the Tcf21. Interestingly, while the S2 E-box was most crucial for Slug inhibition, occupancy of multiple E-box sequences by Tcf21 may achieve a greater degree of inhibition. Previous studies on Slug auto-activation identified binding of the EMT-TF to the S2 E-box; thereby promoter occupancy by Tcf21 may prevent Slug-S2 interaction (18). Further support to these observations is provided by the derepression of Slug promoter following S2 E -box deletion.

While previous reports implicate Tcf21 as a tumor suppressor gene (53), our findings associate nuclear expression of this TF with therapy resistant populations and emphasize a tissue specific activity. Lack of linear correlations between mRNA and protein expression of these TFs along with dynamic alterations in sub-cellular localization highlight immense complexity of the Tcf21-Slug axis and warrant further studies pertaining to additional regulatory networks. Our findings also pose imminent questions regarding the epigenetic landscapes of the E-M spectrum with respect to this axis; cellular compartment specific interacting partners of these TFs that assist their activity; mechanisms linking Tcf21-Slug to specific migratory modes; biochemical evaluation of protein domains responsible for differential sub-cellular localization and TF activity, etc. A retrospective pre-clinical study from our group has further established Tcf21-Slug as potential biomarkers for HGSC tumor stratification and implied the adoption of tumor class-specific therapeutic regimes to effectively target the disease (54). Our report, thus supports this study and presents Tcf21Slug as a crucial axis in HGSC cellular plasticity with genuine clinical implications. 


\section{Acknowledgements}

The authors acknowledge inputs from Ms. Tejaswini Deshpande in bioinformatics analyses for generation of the TF network. We express our gratitude to Dr. S. Mok (M. D. Anderson Cancer Center, Texas, USA) and Prof. Viktor Magdalen (Klinische Forschergruppe der Frauenklinik der TU München) for OV90 and OVMZ6 cell lines. We also extend our gratitude to Prof. Judith Clements (Translational Research Institute, Australia) for providing the OVCA420, PE O14, OVCA432 and CAOV3 cell lines. Matlab_r2013b for analysis of live cell imaging was provided by R.M. Deshmukh (National Centre for Biological Sciences, Bengaluru, India); Dr. A. Karthick (NCCS, Pune, India) and V.K. Vittal (Indian Institute of Science Education and Research, Pune, India) provided expertise in live image processing.

\section{Financial S upport:}

This work was supported by funding to SAB from NCCS, P une (Intramural), and Department of Biotechnology, Government of India, New Delhi (Extramural grants BT/IN/FINNISH/25/S B/2009, BT/Indo-Aus/06/03/2011). Research fellowships were availed as follows - SSV from Council of Scientific and Industrial Research, New Delhi, India; MM from University Grants Commission, New Delhi India; BK from Department of Biotechnology, New Delhi, India, AA from NCCS, Pune, India. 


\section{Data Availability Statement}

The authors declare that all relevant data supporting findings in this study are available within the paper and its supplementary files. Data for quantitative metrics generated from live cell imaging data are available on request.

\section{References}

1. Watson, C.J. et al. (2008) Mammary development in the embryo and adult: a journey of morphogenesis and commitment. Development, 135, 995-1003.

2. Van Amerongen, R. et al. (2012) Developmental stage and time dictate the fate of Wnt/ß-catenin- responsive stem cells in the mammary gland. Cell Stem Cell, 11, 387400.

3. Gonzales, K.A.U. et al. (2017) Skin and Its Regenerative Powers: An Alliance between Stem Cells and Their Niche. Dev. Cell, 43, 387-401.

4. Proia, T.A. et al. (2011) Genetic predisposition directs breast cancer phenotype by dictating progenitor cell fate. Cell Stem Cell, 8, 149-163.

5. Guo, W. et al. (2012) Slug and Sox9 cooperatively determine the mammary stem cell state. Cell, 148, 1015-1028.

6. Varga, J. et al. (2017) Cell plasticity in epithelial homeostasis and tumorigenesis. Nat. Cell Biol., 19, 1133-1141.

7. Nieto, M.A. et al. (2016) E mt: 2016. Cell, 166, 21-45.

8. Tan, T.Z. et al. (2013) Functional genomics identifies five distinct molecular subtypes with clinical relevance and pathways for growth control in epithelial ovarian cancer. EMBO Mol. Med., 5, 983-998.

9. Huang, R.Y.-J. et al. (2013) An EMT spectrum defines an anoikis-resistant and spheroidogenic intermediate mesenchymal state that is sensitive to e-cadherin restoration by a src-kinase inhibitor, saracatinib (AZD0530). Cell Death Dis., 4, e915.

10. Tothill, R.W. et al. (2008) Novel molecular subtypes of serous and endometrioid ovarian cancer linked to clinical outcome. Clin. Cancer Res., 14, 5198-5208.

11. Bell, D. et al. (2011) Integrated genomic analyses of ovarian carcinoma. Nature, 474, 609-615.

12. Yang, D. et al. (2013) Integrated Analyses Identify a Master MicroR NA Regulatory Network for the Mesenchymal Subtype in Serous Ovarian Cancer. Cancer Cell, 23, 186-199.

13. Kanchi, K.L. et al. (2014) Integrated analysis of germline and somatic variants in ovarian cancer. Nat. Commun., 5, 1-14.

14. Gardi, N.L. et al. (2014) Discrete molecular classes of ovarian cancer suggestive of 
unique mechanisms of transformation and metastases. Clin. Cancer Res., 20, 87-99.

15. Domcke, S. et al. (2013) E valuating cell lines as tumour models by comparison of genomic profiles. Nat. Commun., 4, 1-10.

16. Beaufort, C.M. et al. (2014) Ovarian cancer cell line panel (OCCP): Clinical importance of in vitro morphological subtypes. PLoS One, 9,

17. Bapat, S.A. et al. (2005) Stem and Progenitor-Like Cells Contribute to the Aggressive Behavior of Human E pithelial Ovarian Cancer. Cancer Res., 65, 3025-3029.

18. Kumar, B. et al. (2015) Auto-regulation of Slug mediates its activity during epithelial to mesenchymal transition. Biochim. Biophys. Acta - Gene Regul. Mech., 1849, 12091218.

19. Kurrey, N.K. et al. (2009) Snail and slug mediate radioresistance and chemoresistance by antagonizing p53-mediated apoptosis and acquiring a stem-like phenotype in ovarian cancer cells. Stem Cells, 27, 2059-2068.

20. Naik, R.R. et al. (2016) A tumor deconstruction platform identifies definitive end points in the evaluation of drug responses. Oncogene, 35, 727-737.

21. Chen, Y. et al. (2018) Expression of Transcription Factor 21 (TCF21) and Upregulation Its Level Inhibits Invasion and Metastasis in Esophageal Squamous Cell Carcinoma. Med. Sci. Monit., 24, 4128-4136.

22. Mendez, M.G. et al. (2010) Vimentin induces changes in cell shape, motility, and adhesion during the epithelial to mesenchymal transition. FASEB J., 24, 1838-1851.

23. Abid, M.R. et al. (2007) NADPH Oxidase Activity Selectively Modulates Vascular Endothelial Growth Factor Signaling Pathways. J. Biol. Chem., 282, 35373-35385.

24. Roberts, E.W. et al. (2013) Depletion of stromal cells expressing fibroblast activation protein- $\alpha$ from skeletal muscle and bone marrow results in cachexia and anemia. $J$. Exp. Med., 210, 1137-1151.

25. Hennessy, B.T. et al. (2009) Characterization of a naturally occurring breast cancer subset enriched in epithelial-to-mesenchymal transition and stem cell characteristics. Cancer Res., 69, 4116-4124.

26. Rodler, D. et al. (2013) Expression of intermediate filaments in the balbiani body and ovarian follicular wall of the japanese quail (Coturnix japonica). Cells Tissues Organs, 197, 298-311.

27. Takano, S. et al. (2016) Prrx1 is oform switching regulates pancreatic cancer invasion and metastatic colonization. Genes Dev., 30, 233-247.

28. Auersperg, N. (2013) Ovarian surface epithelium as a source of ovarian cancers: Unwarranted speculation or evidence-based hypothesis? Gynecol. Oncol, 130, 246251. 
29. Klotz, D.M. et al. (2017) Cells of origin of ovarian cancer: ovarian surface epithelium or fallopian tube? Arch. Gynecol. Obstet., 296, 1055-1062.

30. Gasparics, Á. et al. (2018) MRTFs-master regulators of E MT. Dev. Dyn., 247, 396404.

31. Acharya, A. et al. (2012) The bHLH transcription factor Tcf21 is required for lineagespecific EMT of cardiac fibroblast progenitors. Development, 139, 2139-2149.

32. Arab, K. et al. (2011) E pigenetic deregulation of TCF21 inhibits metastasis suppressor KISS 1 in metastatic melanoma. Carcinogenesis, 32, 1467-1473.

33. Gooskens, S.L. et al. (2018) TCF21 hypermethylation regulates renal tumor cell clonogenic proliferation and migration. Mol. Oncol, 12, 166-179.

34. Schaeffer, D. et al. (2014) Cellular Migration and Invasion Uncoupled: Increased Migration Is Not an Inexorable Consequence of E pithelial-to-Mesenchymal Transition. Mol. Cell. Biol., 34, 3486-3499.

35. Klymenko, Y. et al. (2017) Heterogeneous Cadherin Expression and Multicellular Aggregate Dynamics in Ovarian Cancer Dissemination. Neoplasia (United States), 19 549-563.

36. Symowicz, J. et al. (2007) Engagement of collagen-binding integrins promotes matrix metalloproteinase-9-dependent E-cadherin ectodomain shedding in ovarian carcinoma cells. Cancer Res., 67, 2030-2039.

37. Mitchell, C.B. et al. (2016) Cooperative cell invasion: matrix metalloproteinasemediated incorporation between cells. Mol. Biol. Cell, 27, 3284-3292.

38. Kamble, S.C. et al. (2013) Stem cell and cancer stem cell games on the ECM field. J. Cancer Stem Cell Res., 1, 1.

39. Sunyer, R. et al. (2016) Collective cell durotaxis emerges from long-range intercellular force transmission. Science, 353, 1157-61.

40. Iwanicki, M.P. et al. (2011) Ovarian cancer spheroids use myosin-generated force to clear the mesothelium. Cancer Discov., 1, 144-157.

41. Dong, Y. et al. (2013) Paclitaxel Resistance and Multicellular S pheroid Formation Are Induced by Kallikrein-Related Peptidase 4 in Serous Ovarian Cancer Cells in an Ascites Mimicking Microenvironment. PLoS One, 8,

42. Aiello, N.M. et al. (2018) E MT Subtype Influences E pithelial Plasticity and Mode of Cell Migration. Dev. Cell, 45, 681-695.e4.

43. E hnert, S. et al. (2012) Transforming growth factor ßlinhibits bone morphogenic protein (BMP)-2 and BMP -7 signaling via upregulation of Ski-related novel protein $\mathrm{N}$ (SnoN): Possible mechanis m for the failure of BMP therapy? BMC Med., 10, 1-11.

44. Zeisberg, M. et al. (2003) BMP-7 counteracts TGF-beta1-induced epithelial-to- 
mesenchymal transition and reverses chronic renal injury. Nat. Med., 9, 964-968.

45. Ng, A. et al. (2015) Ovary and fimbrial stem cells: Biology, niche and cancer origins. Nat. Rev. Mol. Cell Biol., 16, 625-638.

46. Kohan-Ivani, K. et al. (2016) Role of dihydrotestosterone (DHT) on TGF- $\beta 1$ signaling pathway in epithelial ovarian cancer cells. J. Cancer Res. Clin. Oncol, 142, 47-58.

47. Rafehi, S. et al. (2016) TGF $\beta$ signaling regulates E pithelial-mesenchymal plasticity in ovarian cancer ascites-derived spheroids. Endocr. Relat. Cancer, 23, 147-159.

48. Sunde, J.S. et al. (2006) Expression profiling identifies altered expression of genes that contribute to the inhibition of transforming growth factor- $\beta$ signaling in ovarian cancer. Cancer Res., 66, 8404-8412.

49. Cheng, L. et al. (2010) Analysis of chemotherapy response programs in ovarian cancers by the next-generation sequencing technologies. Gynecol. Oncol, 117, 159169.

50. Zhang, S. tao et al. (2016) Identification of key genes associated with the effect of estrogen on ovarian cancer using microarray analysis. Arch. Gynecol. Obstet., 293, 421-427.

51. Berry, N.B. et al. (2008) Ovarian cancer plasticity and epigenomics in the acquisition of a stem-like phenotype. J. Ovarian Res., 1, 8.

52. Xian, W. et al. (2010) The Li-Fraumeni syndrome (LFS): A model for the initiation of p53 signatures in the distal fallopian tube. J. Pathol, 220, 17-23.

53. Maezawa, Y. et al (2014) Loss of the Podocyte-Expressed Transcription Factor Tcf21/Pod1 Results in Podocyte Differentiation Defects and FSGS. J. Am. Soc. Nephrol, 25, 2459-2470.

54. Kamble, S. et al. (2019) Clinical S tratification of High-Grade Ovarian Serous Carcinoma Using a Panel of Six Biomarkers. J. Clin. Med., 8, 330. 


\section{Figure Legends}

Figure 1. Tcf21 and Slug govern cellular phenotypes in HGSC. a. Predicted transcription factor (TF) network of cross-regulatory activities, red arrows / green bars repres ent likely activator / repressor functions respectively; b. Expression profiling of Snai2(Slug),Gli1,Gata4, Peg3,Tcf21,Foxl2 in OVCAR3 and A4 cells by (i) qPCR (heatmap) and (ii) immunoblotting, GAPDH used as endogenous control. Immunoblots represented in the panel are derived from the same experiment and have been processed in parallel; c. Immunostaining of Tcf21,S lug,Ecad,Vim in parental (OVCAR 3 / Tcf2 $1^{\text {native }}, \mathrm{A} 4$ / S lug ${ }^{\text {native }}$ ) and derivative cells (E2 / Tcf $21^{\mathrm{K} / 0}$, D7 / S lug ${ }^{\mathrm{k} / 0}$ and TCF_OE / S lug ${ }^{\mathrm{K} / 0} \mathrm{Tcf} 21^{\mathrm{OE}}$ ), nuclei visualized with Hoechst, Scale bars -

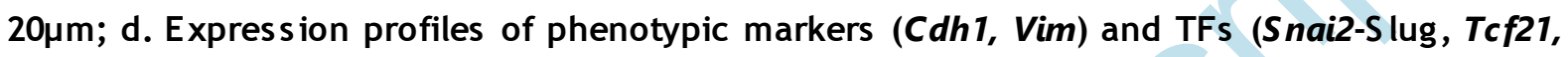
Snail, Zeb1, Zeb2, Twist1, Prrx1) in parental (OVCAR3, A4) and derivative (E2, D7, TCF_OE) cells, normalization with GAPDH; e. Reporter assays to determine the E-cadherin promoter activity in derivative clones (E2, D7, TCF_OE) and parental cells (OVCAR3, A4), luciferase activity normalized to native luciferase vector: $\mathrm{PGL3}$; f. Graphical representation of wound healing efficiency of parental (OVCAR3, A4) and derivative cells (E2, D7, TCF_OE), in the absence and pres ence of serum (upper and lower panels respectively); g. Flow-chart depicting an epithelial- mesenchymal (E-M) axis along which parental (OVCAR 3, A4) and derivative cells (E2, D7, TCF_OE) are placed. Color scheme depicts each of the cell lines represented in preceding panels. All data are representative of experiments performed in triplicate and depicted as mean \pm SEM. ${ }^{*} \mathrm{p}<0.05,{ }^{* *} \mathrm{p}<0.01,{ }^{* * *} \mathrm{p}<0.001$.

Figure 2. Quantitative metrics for resolution of migratory modalities . a. Trajectories depicting migration directionality for parental cell lines (OVCAR 3 / Tcf $21^{\text {native }}, \mathrm{A} 4$ / S lug ${ }^{\text {native }}$ ) and res pective derivatives (E 2 / Tcf $21^{\mathrm{K} / 0}, \mathrm{D} 7 / \mathrm{S} \mathrm{lug}{ }^{\mathrm{K} / \mathrm{O}}$ and TCF_OE / S lug ${ }^{\mathrm{K} / \mathrm{O}} \mathrm{Tcf} 21^{\mathrm{OE}}$ ). P lots repres ent trajectories of 5 randomly selected cells; b. Representative plots depicting mean migratory velocity of parental (OVCAR 3, A4) and derivative cells (E2, D7, TCF_OE). Dis placement and velocity were derived from ' $x$ ' and ' $y$ ' positional co-ordinates detected over a 16 hour duration; $c$. Frequency of nearest neighbours for parental cell lines (OVCAR3, A4) and derivative clones (E2, D7, TCF_OE) at $0 \mathrm{hr}$ (red) and $16 \mathrm{hr}$ (green) time points Dis tribution graphs depict $\mathrm{Nn}$ data for 750 cells . Experiments were performed in the absence / presence of serum. All cells identified in the microscopic field as particles were analyzed for displacement, velocity and $\mathrm{Nn}$. However, only data points inclusive of the ' $X$ ', ' $Y$ ' co-ordinates and $\mathrm{Nn}$ over the entire duration of 16 hours were used for data representation and interpretation; d. Principle component (PC) analys is of time-lapse imaging-based migration data of parental (OVCAR 3, A4) and derivative cells (E2, D7, TCF_OE), PC1 - variance between displacement (Final Y) and velocity vs. neares t neig hbours, PC 2 - variance between dis placement and velocity; color gradient depicts migratory modes, blue-EMT, purple-aCCM, red-pCCM; filled and empty shapes indicate pres ence and absence of serum respectively. 
Figure 3. Tcf21 mediated Slug repression by direct promoter binding. a. Schematic representation of Slug promoter region depicting the location and sequences of $\mathrm{E}$-boxes. Promoter region: $-3 \mathrm{~kb}$ to $+1 \mathrm{~kb}$ from transcription start site (TSS); $\mathrm{b}$. Reporter assays to determine effects of Tcf21 on wild type Slug promoter in A4 cells, luciferase activity normalized to untransfected cells; pEGFP: empty GFP vector, GFP-Tcf21: Tcf21-pEGFPC1 recombinant vector, PGL3: native luciferase vector, PGL3-SlugPR: PGL3 containing Slug promoter; c. Representative EMSA of GST-Tcf21 recombinant protein binding to E-boxes in Slug promoter region in a cell free system, GST protein used as control; d. ChIP-PCR analys is of Tcf 21 binding to E-box elements in Slug promoter in OVCAR 3 cells, IgG used as isotype control, cross-linked fragmented DNA considered as Input. All data are representative of experiments performed in triplicate and depicted as mean \pm SEM. ${ }^{*} \mathrm{p}<0.05,{ }^{* *} \mathrm{p}<0.01,{ }^{* * *} \mathrm{p}<0.001$.

Figure 4. Resolution of the epithelial - mesenchymal phenotypic axis highlights differential functionalities as a function of Tcf21 and Slug localization. a. Expression profiles of Tcf21, Slug, Prrx1 is oforms, Cdh1, Ck19, Vim and FAP in HGSC cell lines at steady states and post serum deprivation normalized with GAPDH. Filled shapes indicate the pres ence of serum and empty shapes indicate that cells were serum starved; b. Representative graphs depicting frequency and intensity of Tcf21 and Slug positive nuclei in HGSC cell lines. Data depicts fluorescence intensities for each transcription factor measured across five random microscopic fields and quantified relative to the signal for Hoechst; c. Repres entative images of suspension culture morphology (Scale bars-200 $\mu$ ); d. Percent cell invasion in Boyden chamber matrigel assay in the abs ence and presence of serum; e. Efficacy of wound healing in the (i) abs ence and (ii) presence of serum; e.iii. Principle component analys is us ed to project segregation of migratory modes in the phenotypic spectrum based on quantitative metrics Final ' $Y$ ', velocity and nearest neighbours - emerging from time-lapse based migration data; color gradient repres ents the migratory modes of each cell line blue-EMT, purple-aCCM, redPCCM; filled shapes indicate the presence of serum and empty shapes indicate that cells were serum starved. All data are representative of experiments performed in triplicate and are depicted as mean + SEM, ${ }^{*} \mathrm{p}<0.05,{ }^{* *} \mathrm{p}<0.01,{ }^{* * *} \mathrm{p}<0.001$.

Figure 5. Tcf21/S lug sub-cellular localization correlates with HGSC wound healing efficacy. a. Representative graphs depicting frequency and intensity of Tcf21 and Slug positive nuclei in HGSC cell lines and derivative clones (E2 / Tcf2 $1^{\mathrm{K} / 0}$, D7 / Slug ${ }^{\mathrm{k} / 0}$ and TCF_OE / S lug ${ }^{\mathrm{K} / 0} \mathrm{Tcf}^{2} 1^{\mathrm{OE}}$ ) exposed to BMP7, TGFB and BMP7+TGFB. Data depicts fluorescence intensities for each transcription factor meas ured across five random microscopic fields and quantified relative to the signal for Hoechst; b. Graphical representation of wound healing efficiency of HGSC cell lines and derivative clones (E2, D7 and TCF_OE) following growth factor treatment as 
indicated; All data are representative of experiments performed in triplicate and depicted as mean \pm SEM. ${ }^{*} \mathrm{p}<0.05,{ }^{* *} \mathrm{p}<0.01,{ }^{* * *} \mathrm{p}<0.001$.

Figure 6. Class-switching in HGSC correlates with therapy induced Slug repression via Tcf21. a PC analys is used to project migratory modes of the different HGSC cell lines following paclitaxel expos ure, PC 1 and PC 2 as described earlier; empty and filled shapes correspond to 0.1\% DMSO and paclitaxel exposure respectively in subsequent figures; $b$. Representative graphs depicting frequency and intensity of Tcf21 and Slug positive nuclei in HGSC cell lines, data depicts fluorescence intensities for each transcription factor measured across five random microscopic fields following expos ure to 0.1\% DMSO (vector control) / paclitaxel (Pac) and quantified relative to the signal for Hoechst; C. ChIP-PCR analys is depicting enrichment of Slug promoter regions in Tcf21 pulldown following paclitaxel exposure of A4 cells. Ig G and DMSO used as isotype and vector control respectively; $d$. Reporter assays to determine effects of Tcf21 on wild type Slug promoter in A4 cells, luciferase activity normalized to untransfected cells. pEGFP: empty GFP vector, GFP-Tcf21: Tcf21-pEGFPC 1 recombinant vector, PGL3: native luciferase vector, pGL3-SlugPR: $p G L 3$ containing Slug promoter, $p G L 3-\triangle S 2-$ SlugPR: PGL3 containing Slug promoter with deleted S $2 \mathrm{E}$-box. All data are repres entative of experiments performed in triplicate and are depicted as mean $\pm \mathrm{SEM}$. ${ }^{*} \mathrm{p}<0.05,{ }^{* *} \mathrm{p}<0.01$, ${ }^{* * *} \mathrm{p}<0.001$. 
Figure 1
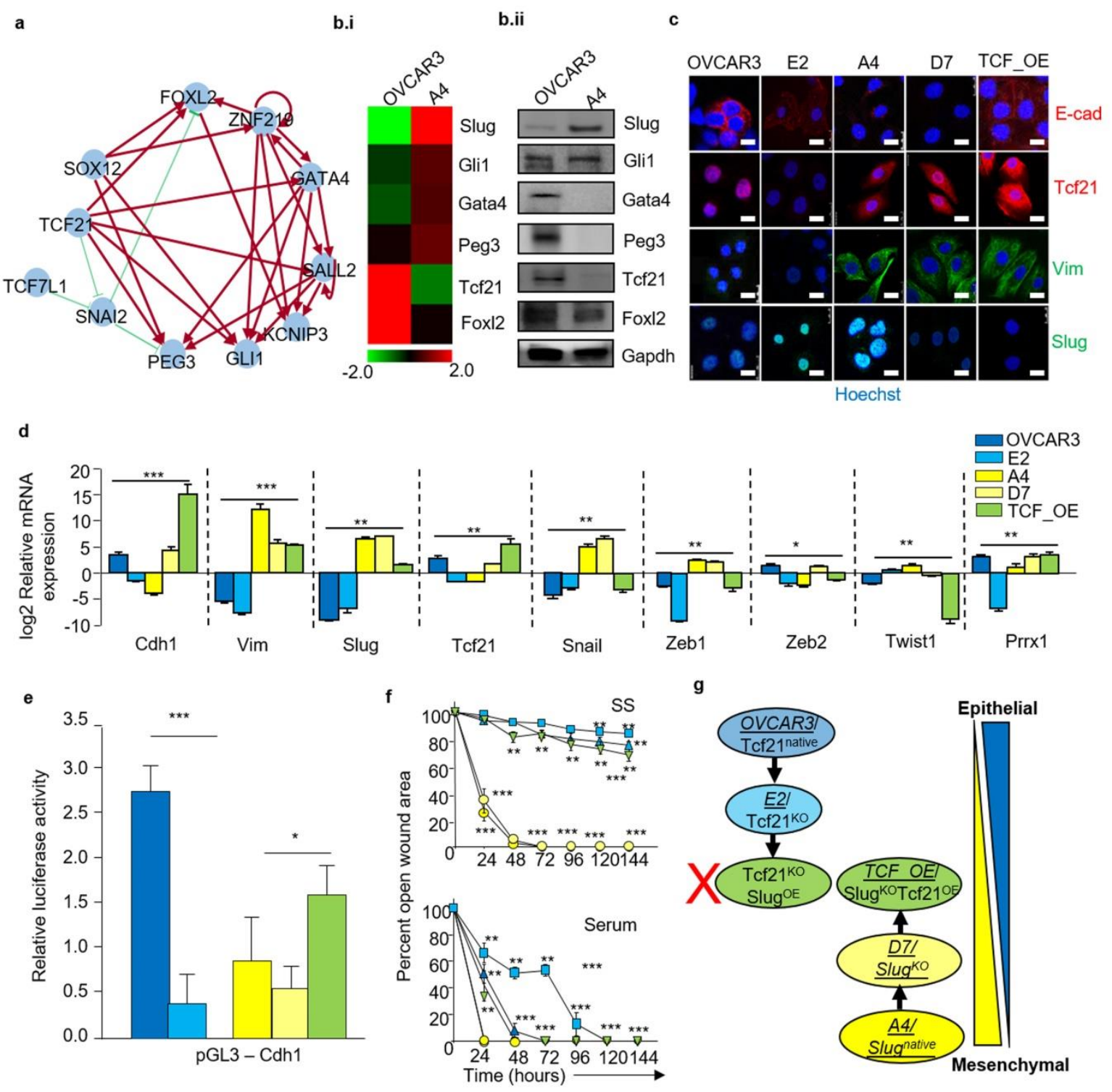
Figure 2

a
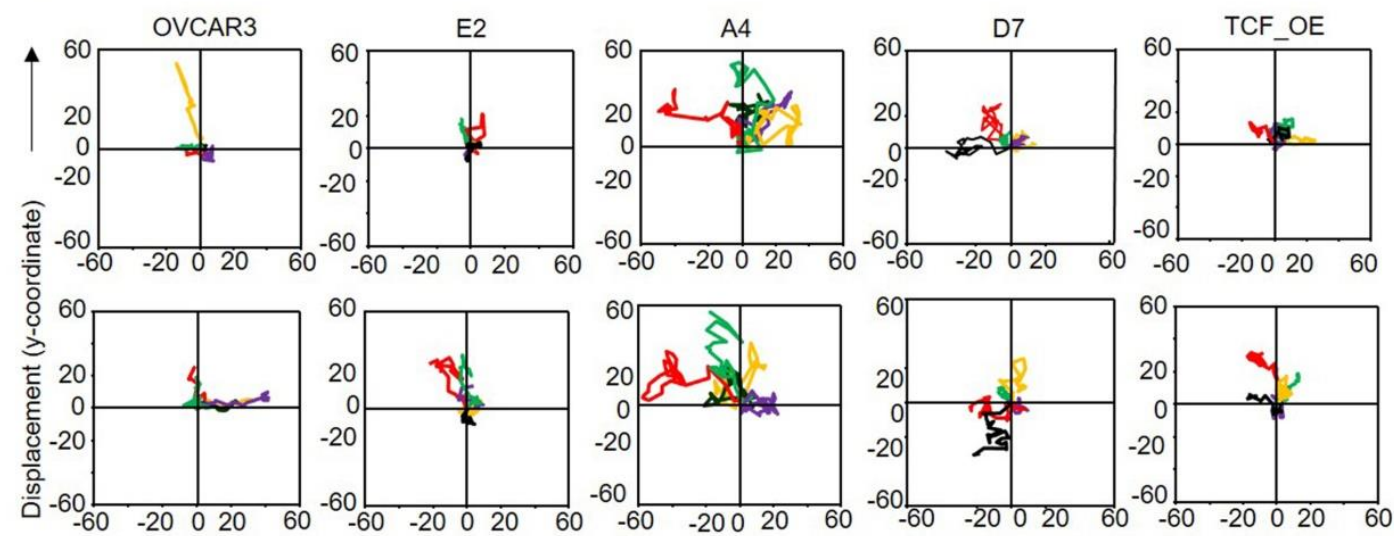

b

Displacement (x-coordinate)

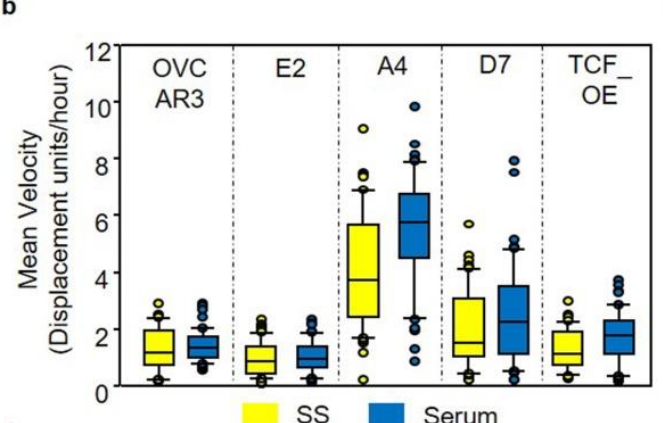

c

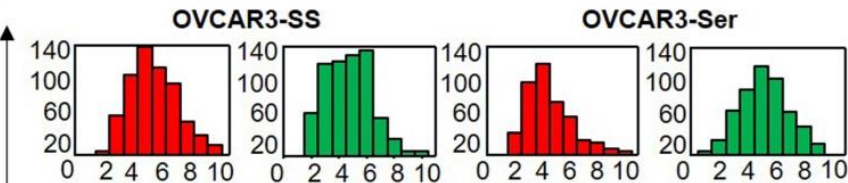

E2-SS

E2-Ser

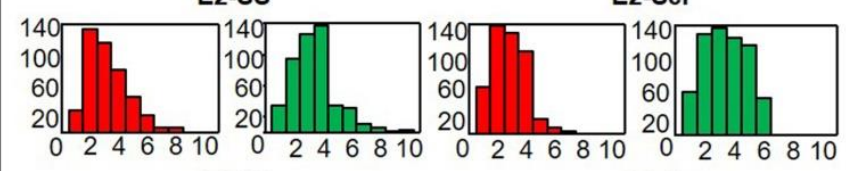

A4-SS

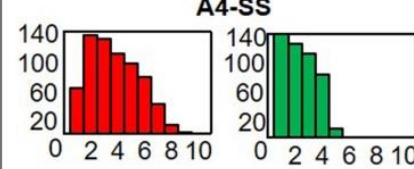

A4-Ser

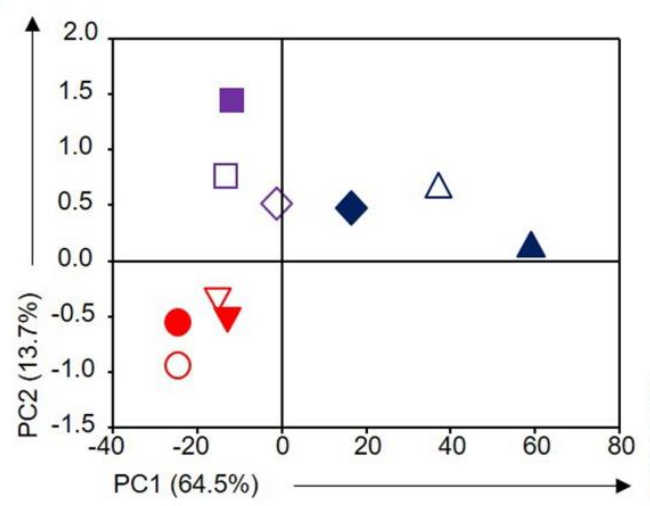

O OVCAR3 $\nabla$ E2 $\square$ TCF_OE $\diamond$ D7 $\triangle$ A4
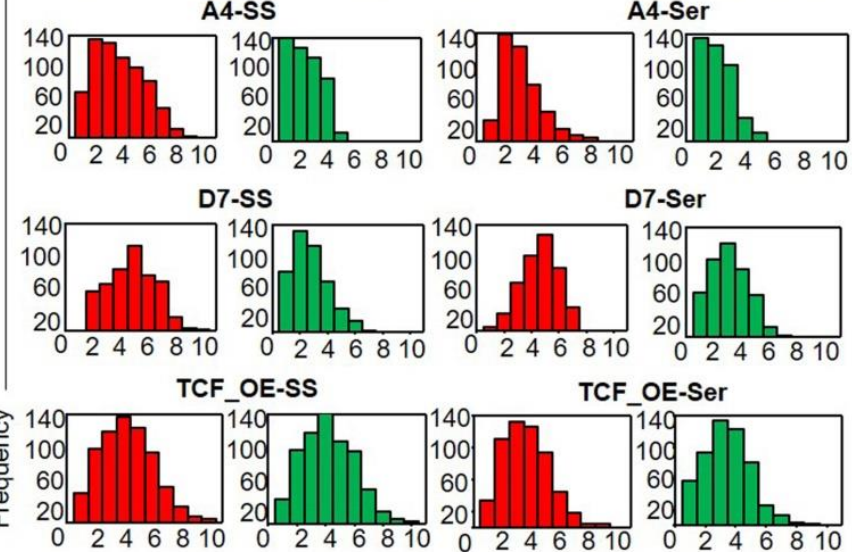

D7-Ser

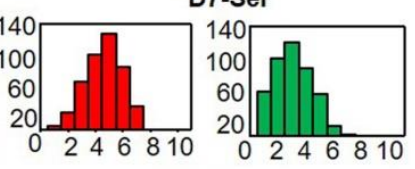

TCF OE-Ser

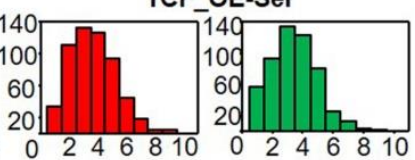

No. of nearest neighbours 
Figure 3

\begin{tabular}{|c|c|c|c|c|c|}
\hline \multirow[b]{2}{*}{$-3 k b$} & \multirow[b]{2}{*}{ s2 } & \multirow[b]{2}{*}{ S3 } & \multicolumn{3}{|c|}{ TSS (0kb) } \\
\hline & & & & $5 \mathrm{~S} 6 \mathrm{~S} 7 \longrightarrow$ & $+1 \mathrm{~kb}$ \\
\hline $\begin{array}{l}\text { E-box } \\
\text { Sequence }\end{array}$ & Position & $\begin{array}{l}\text { E-box } \\
\text { Sequence }\end{array}$ & Position & $\begin{array}{l}\text { E-box } \\
\text { Sequence }\end{array}$ & Position \\
\hline CACCTG & $\begin{array}{l}\text { S1: }(-2346 \text { to }-2341) \text {, } \\
\text { S3: }(-1427 \text { to }-1283) \text {, } \\
\text { S4: }(-1288 \text { to }-1283) \text {, } \\
\text { S7: }(-112 \text { to }-107)\end{array}$ & CAGGTG & S2: $(-1817$ to -1812$)$ & CAGCTG & $\begin{array}{l}\text { S5: }(-267 \text { to }-262) \text {, } \\
\text { S6: }(-229 \text { to }-224)\end{array}$ \\
\hline
\end{tabular}

b

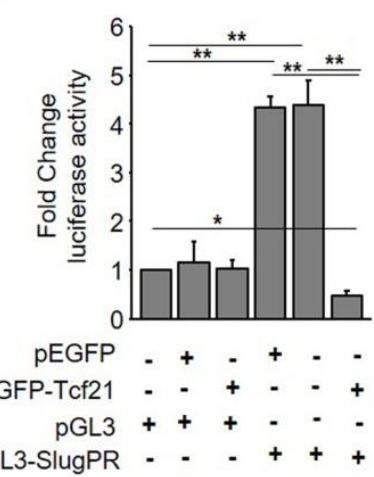

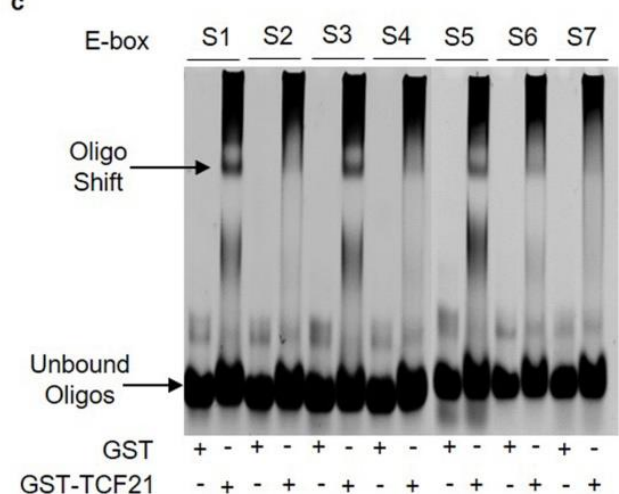

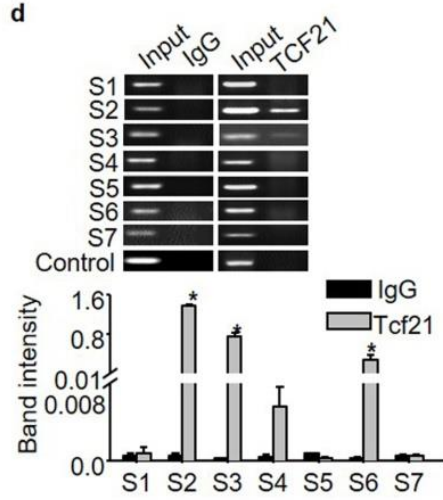


Figure 4

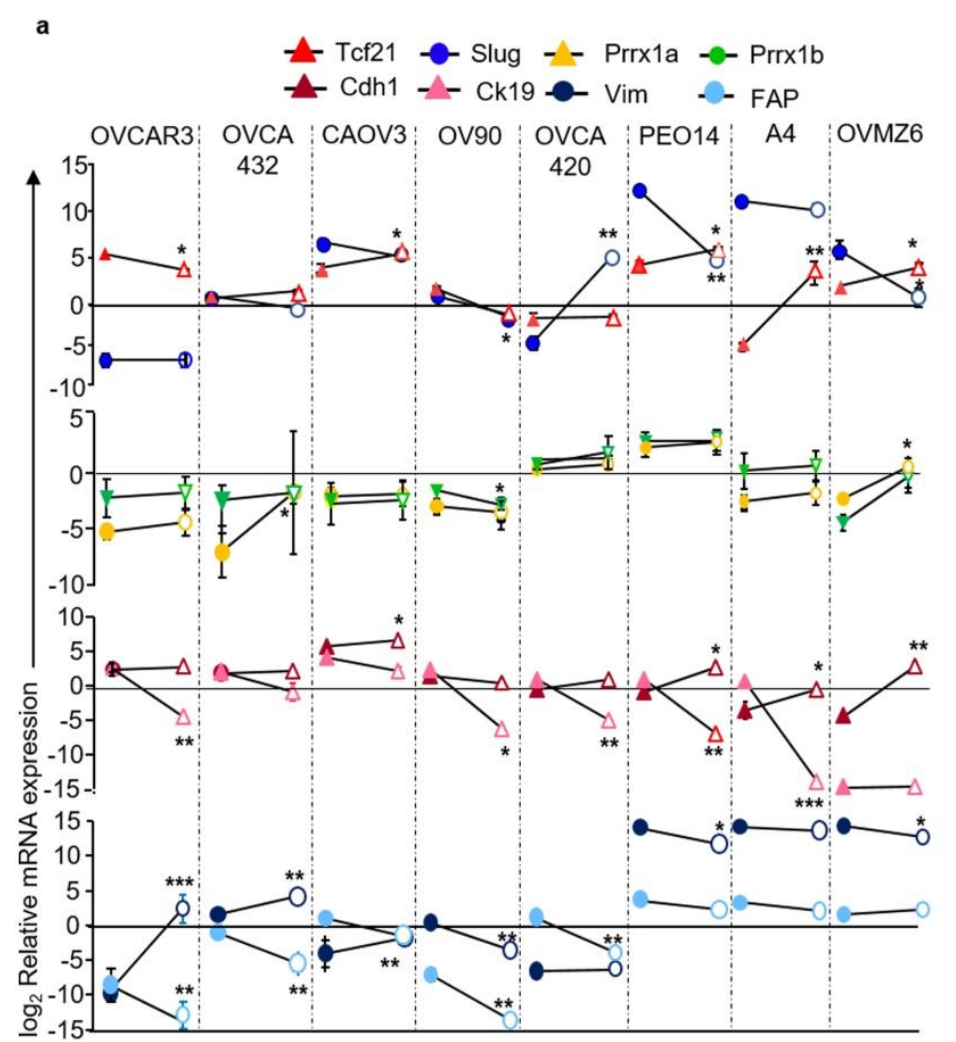

b
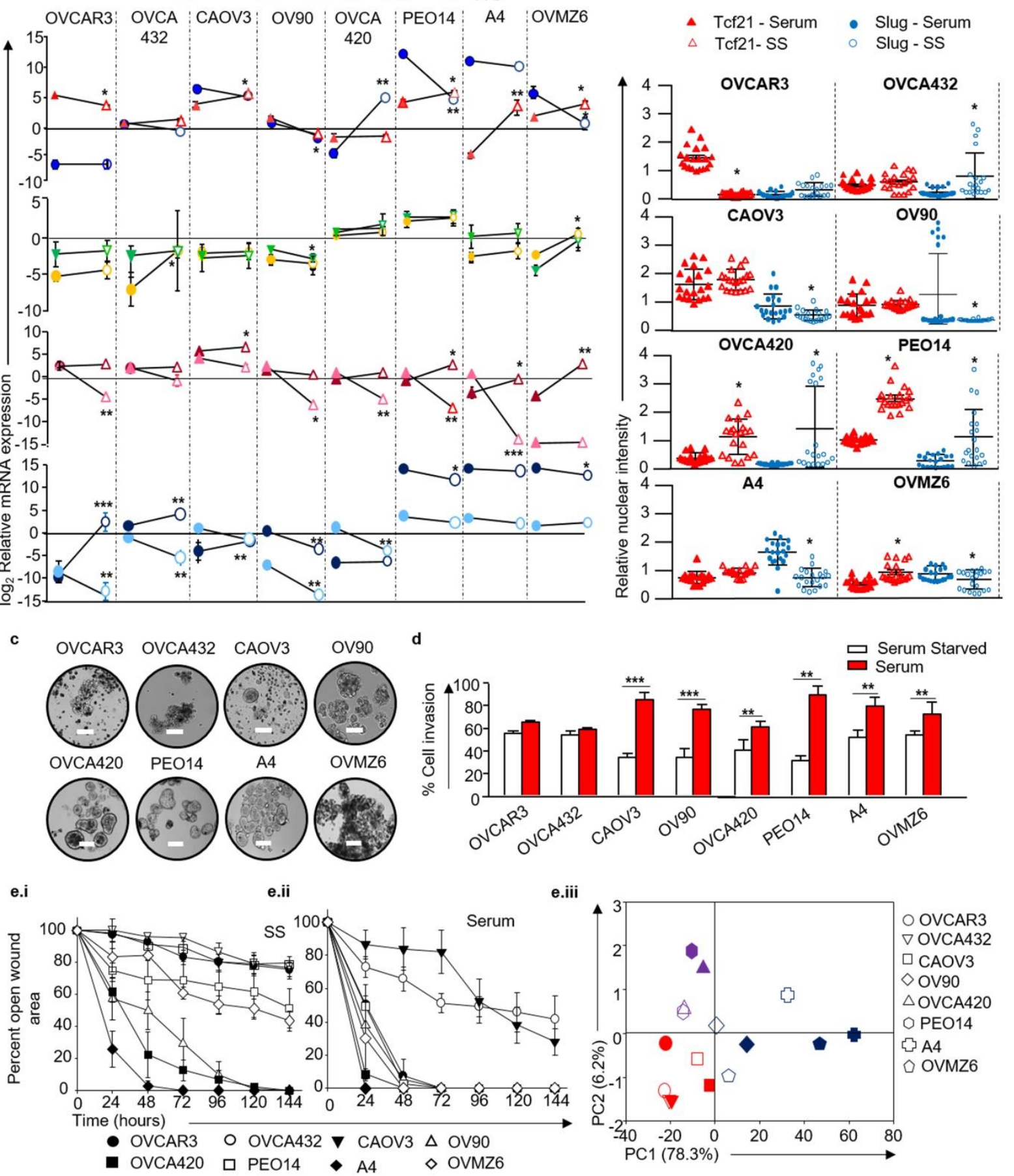
Figure 5

a.

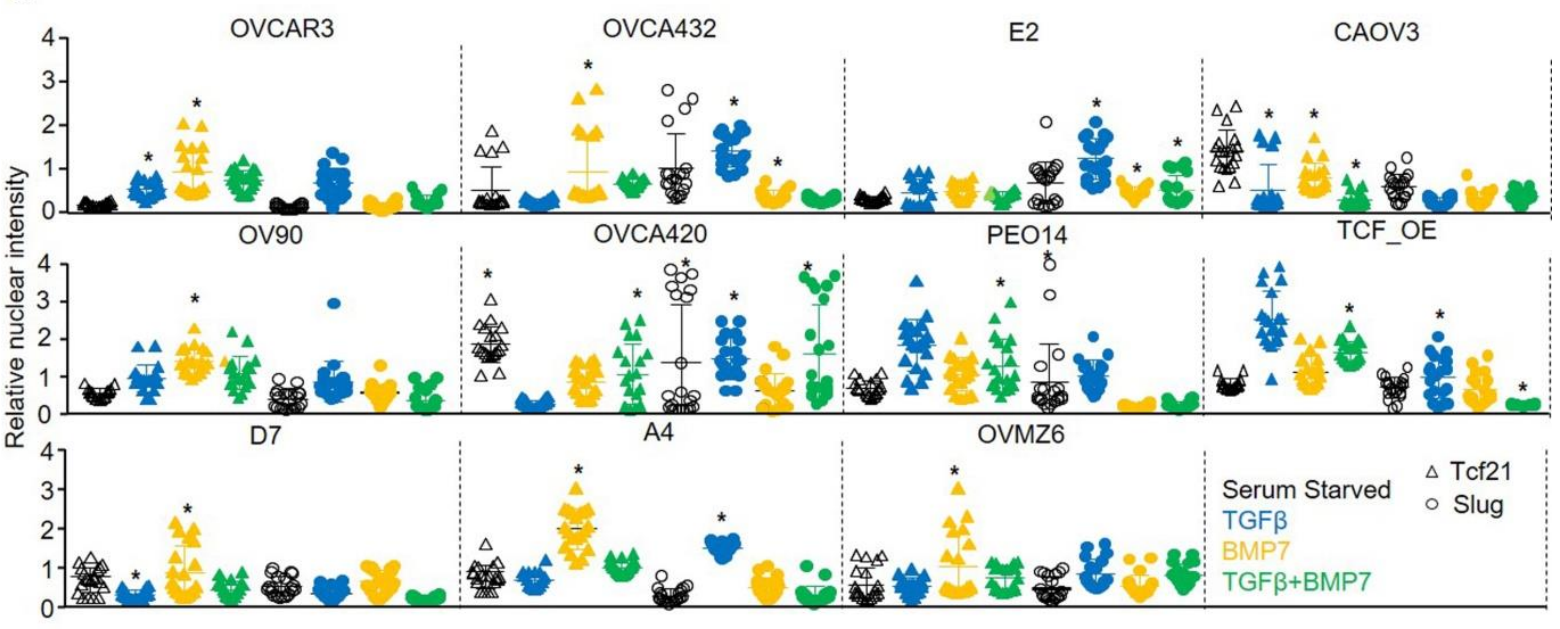

b.

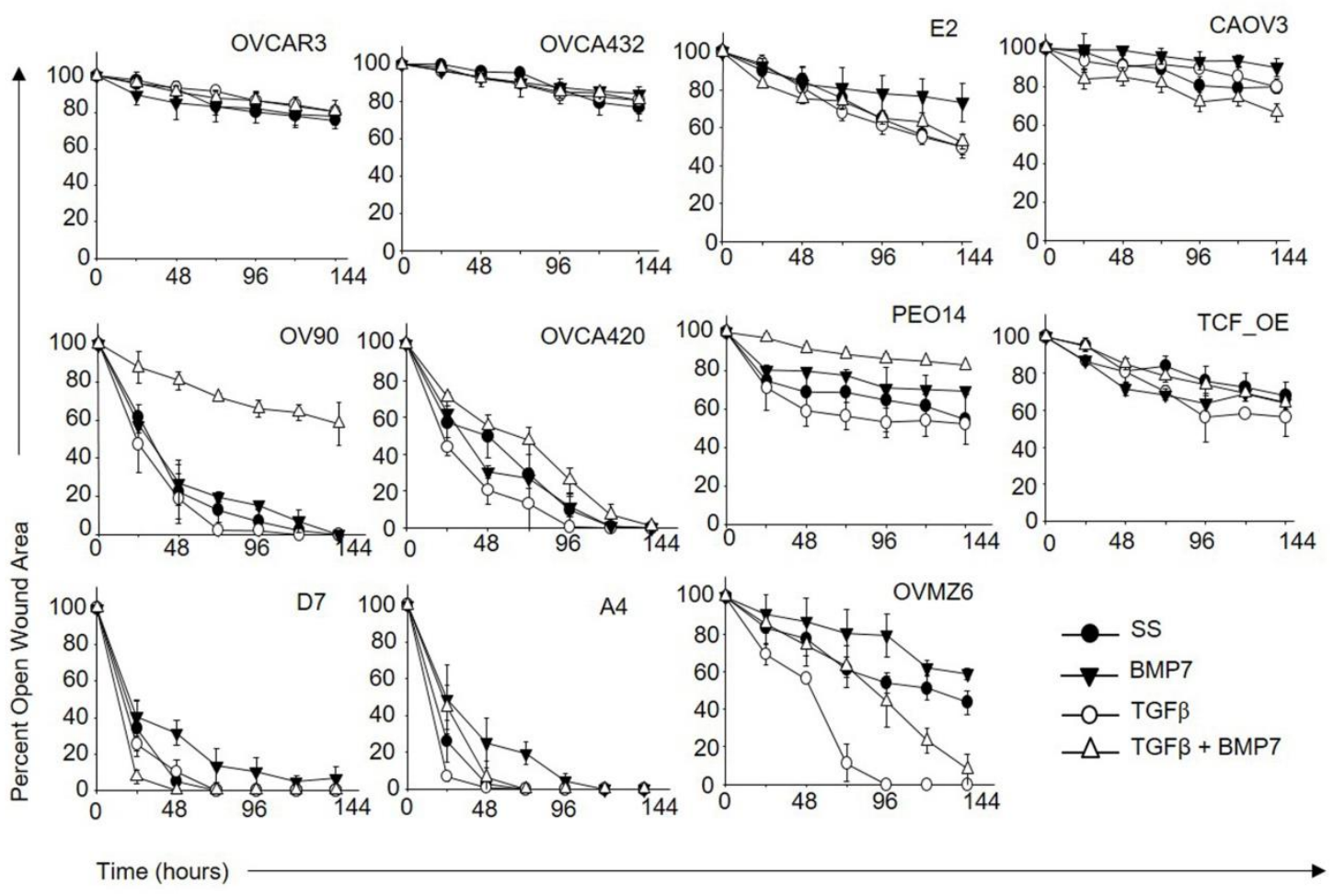


Figure 6

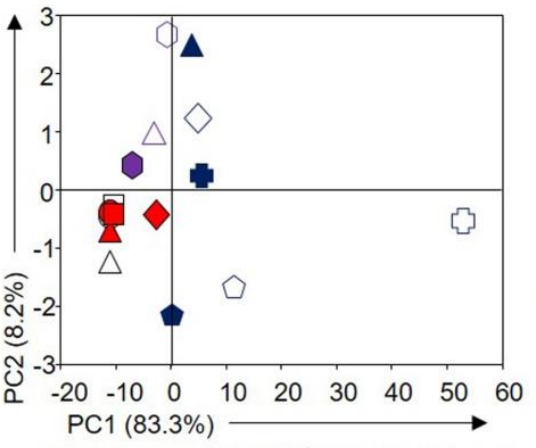

O OVCAR3 $\square$ CAOV3 $\triangle$ OVCA420凸 A4

$\nabla$ OVCA432 $\diamond$ OV90 $\bigcirc$ PEO14 $\triangle$ OVMZ6 b
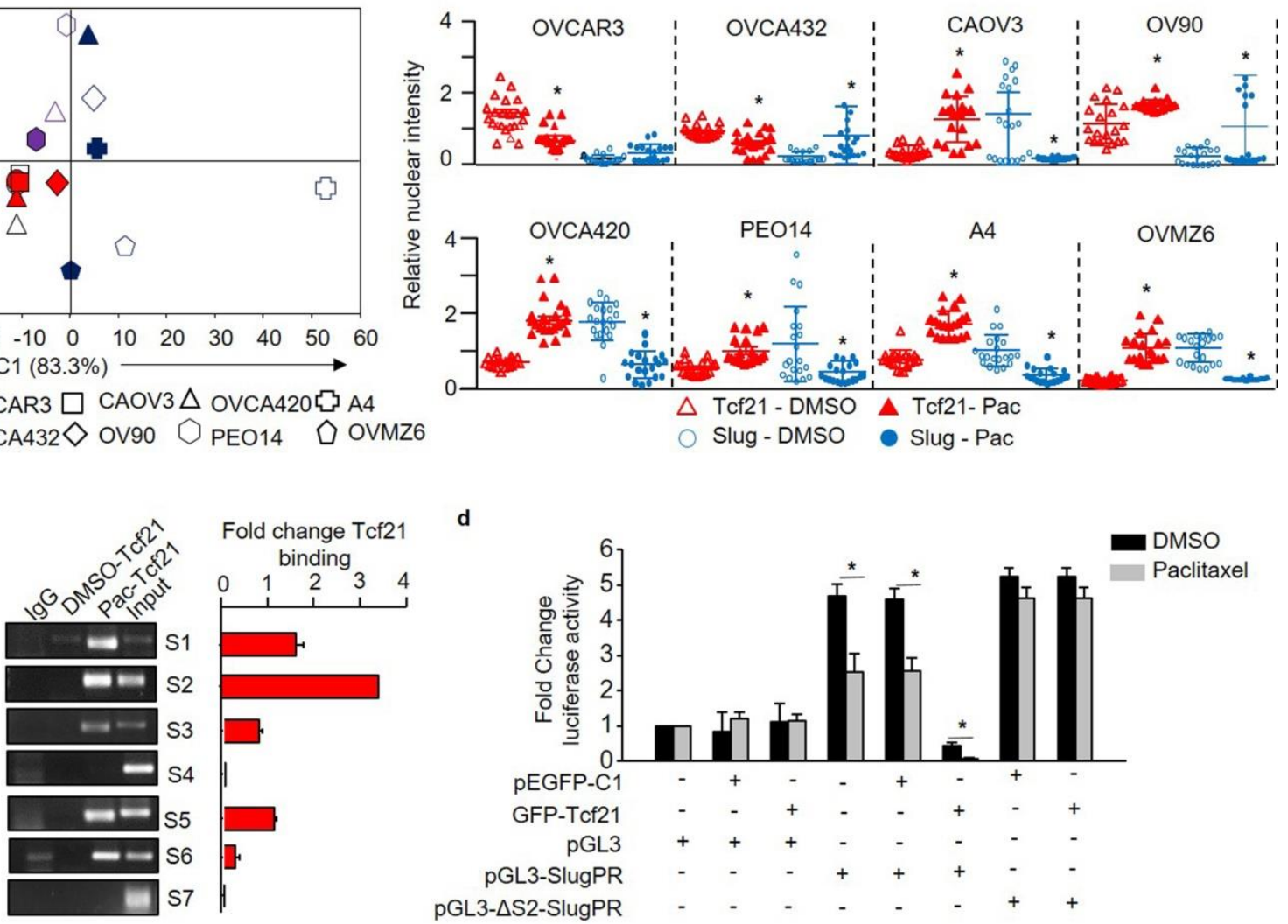